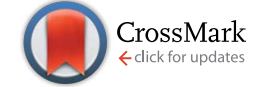

Cite this: RSC Adv., 2017, 7, 9423

\title{
A mechanistic insight into the organocatalytic properties of imidazolium-based ionic liquids and a positive co-solvent effect on cellulose modification reactions in an ionic liquid $\uparrow$
}

\author{
Ryohei Kakuchi, ${ }^{a}$ Ryo Ito, ${ }^{c}$ Shuhei Nomura, ${ }^{a}$ Hadi Abroshan, ${ }^{e}$ Kazuaki Ninomiya, \\ Tomoyuki Ikai, ${ }^{, d}$ Katsuhiro Maeda, ${ }^{\star c d}$ Hyung J. Kim ${ }^{\star e f}$ and Kenji Takahashi*a
}

Received 24th December 2016 Accepted 24th January 2017

DOI: $10.1039 / \mathrm{c} 6 \mathrm{ra2} 8659 \mathrm{c}$

rsc.li/rsc-advances

\begin{abstract}
Detailed insights into the organocatalytic properties of imidazolium-based ionic liquids (Im-ILs) for transesterification of cellulose with isopropenyl acetate (IPA) are presented. According to model transesterification reactions and their computational analysis, acetate anions of Im-ILs play an essential role in the promotion of the reactions. Mechanistic considerations in the optimization of the protocol of IL-catalyzed transesterification reactions have enabled a significant improvement in reaction conditions and a positive co-solvent effect for cellulose modifications in an imidazolium acetate ionic liquid.
\end{abstract}

\section{Introduction}

Triggered by growing importance of sustainability in the chemical industry, biomass-derived chemicals have received extensive scrutiny. ${ }^{\mathbf{1} 2}$ Among many different biomass-based resources, wood-biomass, namely lignocellulose, is one of the most important and obvious candidates as a chemical feedstock because it is the most widespread natural organic material but is not suitable for human consumption as food. Although lignocellulose is known to be composed of three main components, cellulose, hemicellulose, and lignin, cellulose offers the most important chemical resource because of its clear and homogenous chemical structures. For example, its well-defined structure can enable a rational molecular design of the cellulose-based materials. Despite the promising characters of cellulose and its derivatives, their utilization has been minimal owing to their processing difficulties, most notably, their poor

${ }^{a}$ Faculty of Natural System, Institute of Science and Engineering, Kanazawa University, Kakuma-machi, Kanazawa 920-1192, Japan. E-mail: ktkenji@staff.kanazawa-u.ac.jp; Tel: $+81-76-234-4828$

${ }^{b}$ Institute for Frontier Science Initiative, Kanazawa University, Kakuma-machi, Kanazawa 920-1192, Japan

${ }^{c}$ Graduate School of Natural Science and Technology, College of Science and Engineering, Kanazawa University, Kakuma-machi, Kanazawa 920-1192, Japan. E-mail: maeda@se.kanazawa-u.ac.jp

${ }^{d}$ Research Center for Sustainable Energy and Technology, College of Science and Engineering, Kanazawa University, Kakuma-machi, Kanazawa 920-1192, Japan

${ }^{e}$ Department of Chemistry, Carnegie Mellon University, Pittsburgh, Pennsylvania 15213, USA.E-mail: hjkim@cmu.edu

${ }^{f}$ School of Computational Sciences, Korea Institute for Advanced Study, Seoul 02455, Korea

$\dagger$ Electronic supplementary information (ESI) available. See DOI: $10.1039 / \mathrm{c} 6 \mathrm{ra} 28659 \mathrm{c}$ solubility in a wide range of solvents including both aqueous and organic solvents.

Since 2002 when Rogers and his co-workers found that cellulose can be dissolved in ionic liquids (ILs), a unique class of ionic solvents, ${ }^{3}$ ILs have received increasing interests in biomass-related science and technology., ${ }^{\mathbf{4} 5}$ Due to their attractive properties, in particular, large flexibility in their design, ILs with tailored functionalities were designed and synthesized. ${ }^{6-12}$ Among different functionalities that can be embedded in ILs, the incorporation of organocatalytic attributes is attracting a rapidly increasing attention in synthetic chemistry. ILs thus tailored can offer dual functionalities by acting simultaneously as a solvent for reactants and a catalyst for organic transformation reactions. ${ }^{13}$ In view of ILs' advantage in dissolution of cellulose ${ }^{14-19}$ such tailored ILs have been gradually integrated into cellulose related science. Recently, some of us reported that 1-ethyl-3-methylimidazolium acetate (EmimOAc) can serve not only as a facile solvent for cellulose but also as an activating reagent for a transesterification reaction of cellulose hydroxy groups with isopropenyl acetate (IPA) as an ester donating reagent (Scheme 1). ${ }^{20}$ The synthetic protocol employed enabled facile and promising transesterification of cellulose without any additional catalysts and activating reagents. However, the

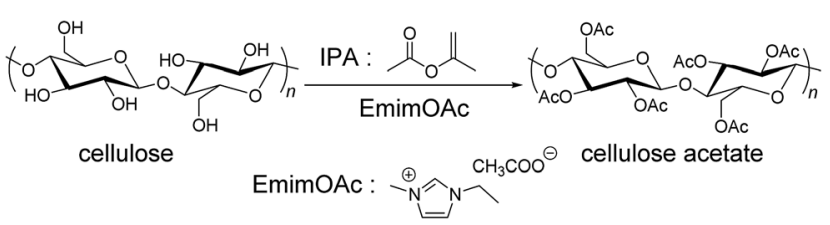

Scheme 1 Schematic representation of the transesterification reaction of cellulose in EmimOAc. 
reaction required fairly large reactant loadings, compared to acylation reactions with activated chemical reagents. This limits the applicability of the protocol in the field of synthetic polymer chemistry. Therefore, the improvement on reaction conditions through an optimization of the synthetic protocol is clearly needed for practical applications.

In order to optimize the above-mentioned synthetic protocol, ${ }^{20}$ a precise understanding of roles played by imidazolium-based ionic liquids (Im-ILs) in the transesterification reactions is needed. Such a rational optimization based on a detailed mechanistic understanding could help to realize, e.g., greener conditions for cellulose modification reactions. The catalytic activities of Im-ILs in a range of different reactions were proposed to be effected via (1) the spontaneous generation of N-heterocyclic carbenes (NHCs) ${ }^{\mathbf{2 1 , 2 2}}$ from parental $\mathrm{ILS}^{23-25}$ or (2) cooperative molecular recognition either by anions or by both the cations and anions of the ILs. ${ }^{26-28}$ Another possibility that should be considered is the nucleophilic assistance by anions of the ILs. Herein, we investigate the ILs' roles and obtain molecular insight into the reaction mechanism by employing a model reaction system. On the basis of this understanding, we rationally optimize the transesterification reaction processes. In this article, we present (1) a mechanistic insight into the roles of Im-ILs for transesterification reactions obtained by a combined experimental and a computational study and (2) a positive co-solvent effect on the Im-ILs catalyzed transesterification reactions of cellulose in Im-ILs based on a precise understanding of the transesterification reaction mechanism.

\section{Results and discussions}

\section{Organocatalytic properties of Im-ILs; analysis of catalytic roles of Im-ILs}

While EmimOAc acts as an efficient solvent for cellulose and also triggers transesterification of cellulose with IPA without any additional activators and catalysts as mentioned above, ${ }^{20}$ dissolution of cellulose in differing environments is needed for a detailed study of the IL's catalytic roles per se. However, insolubility of cellulose in common organic and aqueous solvents makes it difficult to investigate this aspect and thus the mechanism of IL-activated transesterification reaction of cellulose. In order to facilitate the mechanistic analysis, we instead studied a more amenable reaction system by employing 2-phenylethyl alcohol (2-PA) and IPA as a nucleophile and an ester donating reagent, respectively. To test if this can provide a good model reaction system for cellulose, transesterification reaction of IPA and 2-PA was first carried out under $\mathrm{Ar}$ atmosphere at $80{ }^{\circ} \mathrm{C}$ in the presence of a catalytic amount of EmimOAc with $[2-\mathrm{PA}] /[\mathrm{IPA}] /[$ EmimOAc $]=1 / 1.5 / 0.1$. Transesterification of 2-PA and IPA occurred efficiently and generated 2-phenetyl acetate as a product with 63\% conversion in 16 hours, confirming that the transesterification process was an indeed organocatalytic reaction and not an activated (or mediated) reaction (run 1, Table 1). ${ }^{29}$ Next, the effect of anions on transesterification of 2-PA was examined. The results in Table 1 indicate that ILs with weakly basic anions tend to lose their
Table 1 Effect of anion structures on the transesterification reaction of 2-PA and IPA catalyzed by Im-ILs with diverse anions ${ }^{a}$

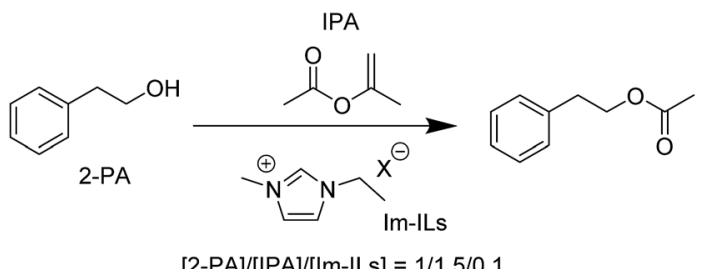

$[2-\mathrm{PA}] /[\mathrm{IPA}] /[\mathrm{Im}-\mathrm{ILs}]=1 / 1.5 / 0.1$

\begin{tabular}{llcl}
\hline Run & Anion $\left(\mathrm{X}^{-}\right)$ & $\mathrm{p} K_{\mathrm{a}}{ }^{b}$ & Conv. $^{c}(\%)$ \\
\hline 1 & $\mathrm{CH}_{3} \mathrm{COO}^{-}$ & 12.3 & 63 \\
2 & $\mathrm{Cl}^{-}$ & 1.8 & 1 \\
3 & $\mathrm{Br}^{-}$ & 0.9 & 1 \\
4 & $\mathrm{CF}_{3} \mathrm{COO}^{-}$ & 3.45 & 2
\end{tabular}

${ }^{a}$ The reaction conditions for transesterification of PA and IPA are as follows; neat condition; the reaction was conducted for 16 hours; $\mathrm{Ar}$ atmosphere. ${ }^{b}$ The $\mathrm{p} K_{\mathrm{a}}$ values in DMSO. ${ }^{30}{ }^{c}$ Determined by ${ }^{1} \mathrm{H}$ NMR in $\mathrm{CDCl}_{3}$.

catalytic reactivity. This clearly demonstrates that the anion basicity plays a crucial role in the organocatalytic properties of Im-ILs. Nevertheless, this finding does not offer any decisive insight on the mechanism for the IL-catalyzed transesterification reaction. In fact, the catalytic activity enhancement with anion basicity observed here could rationalize any of the three mechanisms mentioned above, i.e., (1) catalytic reaction by NHCs generated spontaneously from parental ILs, (2) cooperative molecular recognition by IL ions, or (3) nucleophilic assistance by IL anions. We thus performed additional experiments, as well as DFT calculations, which we turn to next.

The key question we tackled is whether or not carbene species plays a crucial catalytic role in the transesterification processes. The proton at the C-2 position of imidazolium cations is rather acidic, so that its transfer to a base - if it occurs - generates carbene. It is widely conjectured that carbene thus produced participates directly in many reactions, such as benzoin condensation reactions, ${ }^{24,31}$ trapping reactions, ${ }^{23}$ and $\mathrm{CO}_{2}$ chemisorption, ${ }^{32,33}$ in Im-ILs featuring basic anions such as acetate. However, this is controversial in that NHCs have not been detected experimentally in many neat Im-ILs. ${ }^{\mathbf{2 3 , 2 4 , 3 1}}$ Furthermore, according to a recent theoretical work on $\mathrm{CO}_{2}$ capture, the generation of NHC from EmimOAc is highly unlikely in a polar environment due to NHC's instability. ${ }^{34}$

To gain insight into the catalytic mechanism of our transesterification reaction system and more broadly into the controversial issues surrounding carbene in Im-ILs, we modified the chemical structure of Im cations in a systematic and controlled way and analyzed its influence on transesterification kinetics. By employing 1,3-bis(2,6-diisopropylphenyl) imidazolium acetate (BiPrimOAc) and 1-ethyl-3-methyl benzimidazolium acetate (EMBnimOAc), the effect of substitutions at the $\mathrm{N}-1, \mathrm{~N}-3, \mathrm{C}-4$ and $\mathrm{C}-5$ positions was first investigated. Comparison of the results in Table 2 (runs 1, 5, and 6) indicates that the chemical modifications at the N-1, N-3, C-4 and C-5 positions of Im cations have little influence on the catalytic 
Table 2 Effect of counter-cation structures on the transesterification reaction of 2-PA and IPA catalyzed by acetate salts ${ }^{a}$
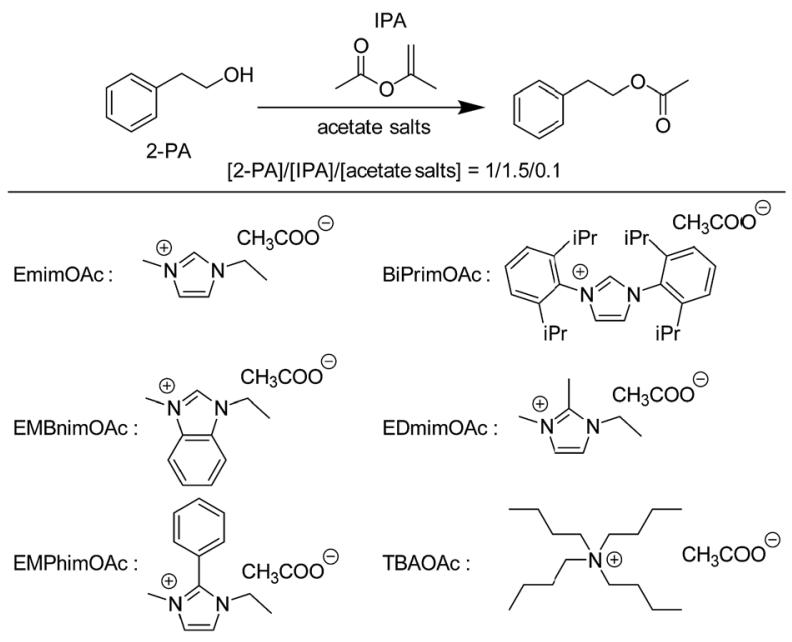

\begin{tabular}{lll}
\hline Run & Acetate salts & Conv. $^{b}(\%)$ \\
\hline 1 & EmimOAc & 63 \\
5 & BiPrimOAc & 61 \\
6 & EMBnimOAc & 49 \\
7 & EDMimOAc & 29 \\
8 & EMPhimOAc & 22 \\
9 & TBAOAc & 27
\end{tabular}

${ }^{a}$ The reaction conditions for transesterification of 2-PA and IPA are as follows; neat condition; the reaction was conducted for 16 hours; $\mathrm{Ar}$ atmosphere. ${ }^{b}$ Determined by ${ }^{1} \mathrm{H}$ NMR in $\mathrm{CDCl}_{3}$.

activity of Im-ILs toward transesterification. Next, the effect of substitution at the $\mathrm{C}-2$ position was examined. If the reaction is mainly catalysed by carbenes, chemical capping of the C-2 position of Im-ILs should lead to a major loss of the catalytic activity of ILs. As demonstrated in run 7 (Table 2), transesterification between 2-PA and IPA was catalysed by 1-ethyl-2,3dimethyl imidazolium acetate (EDmimOAc), whose C-2 position was masked with methyl group. While this result rationalizes the crucial role played by anions in the catalytic reactions, spontaneous generations of $\mathrm{N}$-heterocyclic olefins (NHOs) from parental ILs could not be excluded completely. Recently, NHOs were revealed to behave as an efficient organocatalyst for ringopening polymerization of epoxides and lactones. ${ }^{35,36}$ Therefore, the C2-phenyl capped Im-IL, 1-methyl-2-phenyl-3-ethyl imidazolium acetate (EMPhimOAc), was employed as a control compound, where deprotonation reactions from imidazolium skeleton could be completely shut out. As clearly demonstrated, a facile organocatalytic transesterification reaction was confirmed in the presence of EMPhimOAc, showing that EMPhimOAc can still act as an efficient organocatalyst for the transesterification reaction (run 8, Table 2). In addition, tetrabutylammonium acetate (TBAOAc) also catalyzed transesterification of 2-PA and IPA with a $27 \%$ conversion (run 9, Table 2). These experimental data provide clear evidence that the organocatalytic activity of Im-ILs in transesterification reactions is mainly due to the nucleophilic assistance by anions of the ILs.
This is consistent with a recent theoretical study that the generation of NHC's in 1-alkyl-3-methylimidazolium acetate is very unlikely due to NHC's instability in a polar environment. ${ }^{34}$

\section{Computational analysis of organocatalytic properties of Im- ILS}

DFT calculations were performed to understand the experimental results on the transesterification reactions (Fig. 1 and $\mathrm{S} 3 \dagger$ ), in particular, the roles played by ILs. For efficient calculations, the imidazolium cation and the alcohol were modelled as 1,3-dimethylimidazolium (DMIM) and methanol, respectively. We first investigated the reaction pathway in the absence of the ILs. DFT results indicate that barrier height for the reaction is rather high, $30.9 \mathrm{kcal} \mathrm{mol}^{-1}$ (Reac $\rightarrow 1 \mathrm{TS}_{1}$, Fig. 1), which explains no conversion in the absence of catalysts. Next, we studied the effect of the IL anion, acetate, on the energy profile of the reaction. As illustrated in Fig. 1, acetate interacts strongly with the proton of methanol's hydroxy group $\left(26.5 \mathrm{kcal} \mathrm{mol}^{-1}\right.$, Reac $\rightarrow 1$ Int $_{1}$ ). This leads to a significant elongation of $\mathrm{O}-\mathrm{H}$ bond by $0.04 \AA$, which in turn changes the partial charge of the alcohol's oxygen from -0.60 to -0.70 (Fig. S1†). We would thus expect that the alcohol's oxygen becomes "activated" and hence enabled to react with carbonyl group of the ester. As expected, DFT predicts that the barrier height for the reaction between the $\left[\mathrm{CH}_{3} \mathrm{OH} \cdots \mathrm{OAc}\right]^{-}$complex and ester is $2.6 \mathrm{kcal} \mathrm{mol}^{-1}$, markedly lower than $30.9 \mathrm{kcal} \mathrm{mol}^{-1}$ in the absence of the anion above $\left(1 \mathrm{Int}_{1} \rightarrow 1 \mathrm{TS}_{2}\right.$, Fig. 1). This result is in good agreement with the experimental observation made above, i.e., an increase in the basicity of IL anions leads to a higher reaction conversion (Table 1 ). The transition state $1 \mathrm{TS}_{2}$ eventually converts to products through an exothermic step of $1.1 \mathrm{kcal} \mathrm{mol}^{-1}\left(1 \mathrm{TS}_{2} \rightarrow\right.$ Pro).

Turning to imidazolium cations, we mainly focus on the acidic hydrogen at the $\mathrm{C}-2$ position, which tends to interact

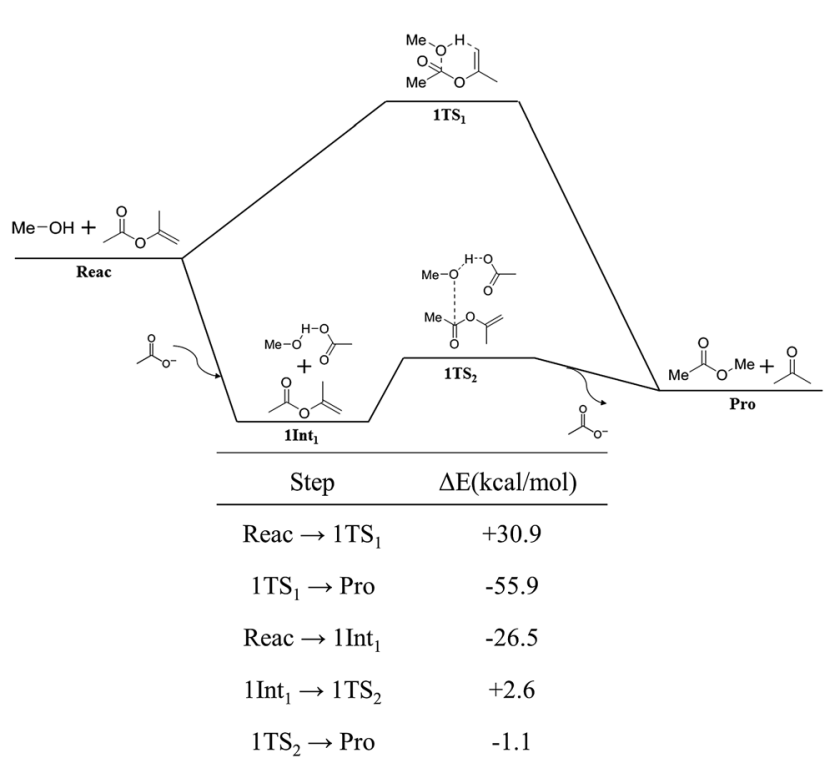

Fig. 1 Proposed reaction mechanism 1 for transesterification reactions in the absence (upper pathway) and presence (lower pathway) of acetate anion of the ionic liquids along with the calculated energies for different elementary steps. 
strongly with negatively charged sites compared to other ring or alkyl chain hydrogen atoms. It plays a significant role in transesterification kinetics (run 1 vs. runs 7 and 8, Table 2), whereas chemical substitutions at other positions have only a minor effect as noted above. DFT predicts that the interaction of the acidic proton at the $\mathrm{C}-2$ position of DMIM with the carbonyl oxygen of the ester induces a pronounced conformational deformation of the ester's skeleton - i.e., the latter changes from a planar to a twisted geometry (Fig. S2 in the ESI $\dagger$ ). As such, the Im-ester interaction via the acidic proton and carbonyl oxygen is expected to exert a considerable influence on the reaction energetics (Fig. S3†). According to DFT calculations, this interaction reduces the activation energy for transesterification by 2.8 kcal mol ${ }^{-1}$ (2Int $\rightarrow$ 2TS, Fig. S3 $\dagger$ ), compared to that in its absence (Reac $\rightarrow 1 \mathrm{TS}_{1}$, Fig. 1 ). This result is in concert with our experimental findings above (run 5 vs. 8, Table 2). It is also noteworthy that the effect of the acidic proton is considerably weaker than that of the anion, as evidenced by the DFT results (2.8 vs. $28.3 \mathrm{kcal} \mathrm{mol}^{-1}$ in the barrier height reduction) and confirmed by experimental results (Tables 1 and 2).

In view of the experimental and computational results discussed above, we propose a mechanism for the transesterification reaction of alcohol and ester catalyzed by Im-ILs as follows (Fig. 2): first, the $\mathrm{O}-\mathrm{H}$ group of alcohol is activated by acetate anions, while the carbonyl group of ester is activated by interacting with the acidic proton of the ILs. Next, alcohol and ester thus activated couple to form a transition state, characterized by a strong $\mathrm{C} \cdots \mathrm{O}$ interaction between the two. Eventually, this activated complex becomes a product with the conversion of the $\mathrm{C} \cdots \mathrm{O}$ interaction to a new ester bond and the release of ions for the regeneration of the catalytic species (Fig. 2).

\section{A positive co-solvent effect on cellulose modification reactions in EmimOAc}

In the transesterification mechanism proposed above, the catalytic behaviour of the Im-ILs is mainly due to nucleophilic assistance by IL anions of alcoholic species. Therefore the

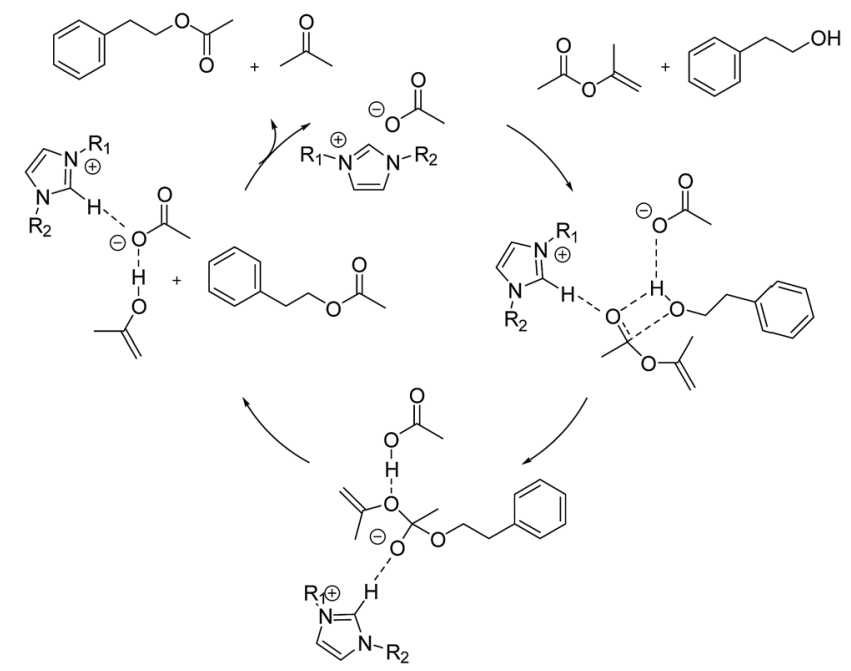

Fig. 2 Plausible mechanism for the transesterification reaction of 2PA and IPA catalyzed by imidazolium acetates. addition of an aprotic solvent, such as DMSO, as a co-solvent would have a large influence on the reactions. Aprotic polar solvents are known to coordinate strongly with cationic species. ${ }^{37,38}$ Resulting solvation of cations would facilitate the dissociation of ion pairs and thus generation of "naked" anions, ${ }^{37,38}$ which would promote the reactivity of alcoholic species. With this expectation, an optimization of cellulose modification reactions in ILs was targeted. From synthetic chemistry perspective, the goal was to reduce the amount of ILs needed for reactions while maintaining high conversions. We first studied the model reaction system to check whether or not the catalytic promotion via aprotic co-solvents could be realized. As expected, transesterification reaction of 2-PA and IPA was accelerated with the addition of DMSO, compared to transesterification in the absence of a co-solvent (Fig. 3). Specifically, the EmimOAc-catalyzed transesterification in the presence of DMSO reached a $42 \%$ conversion of 2 -PA in 7 hours at $80{ }^{\circ} \mathrm{C}$, while that in the absence of DMSO yielded only an $18 \%$ conversion during the same period. This experimental result confirms that an addition of DMSO as a co-solvent accelerates the IL-catalyzed transesterification reactions.

Encouraged by the positive co-solvent effect on the transesterification reactivity, we finally aimed to optimize the cellulose modification reactions in ILs. In a very recent study, it was found that transesterification reactions of cellulose with IPA in EmimOAc yielded a smooth conversion to cellulose acetates with a high degree of substitution (DS). ${ }^{20}$ One notable drawback of the synthetic protocol employed there is a need to use ILs as a solvent, which requires a large amount of ILs. This places a significant restriction on its practical application from both synthesis and cost perspective because ILs are very viscous and
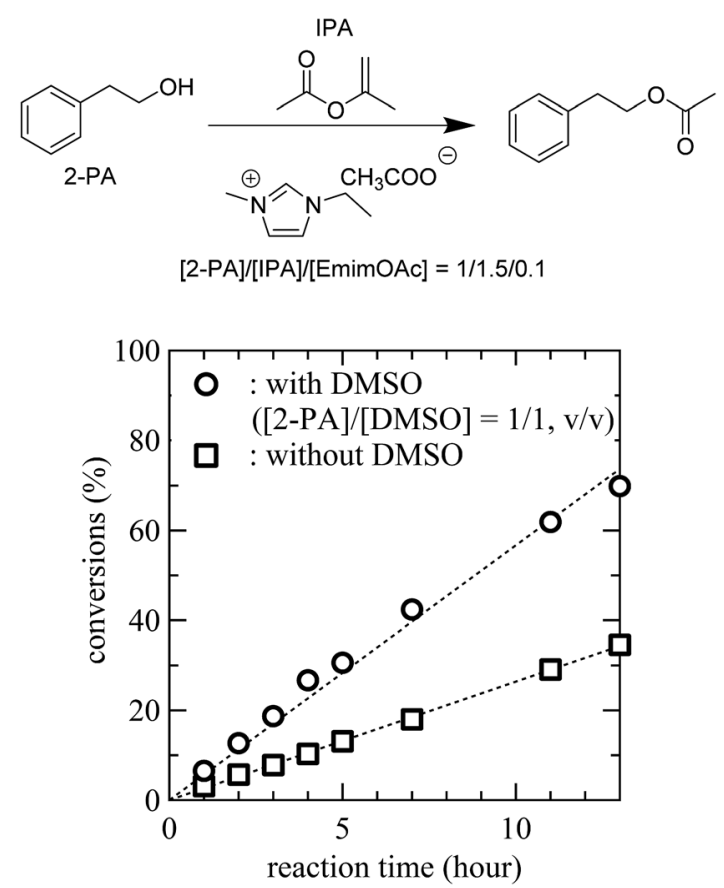

Fig. 3 Effect of DMSO addition on the transesterification reaction of the 2-PA and IPA catalyzed by EmimOAc (line; guidance). 
Table 3 Effect of amount of ionic liquids on the transesterification reaction of cellulose and IPA catalyzed by EmimOAc ${ }^{a}$

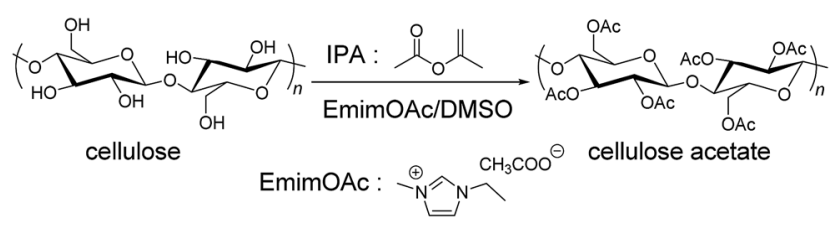

\begin{tabular}{|c|c|c|c|c|}
\hline Run & EmimOAc (mg) & $\begin{array}{l}\text { EmimOAc/cellulose } \\
\text { (wt ratio) }\end{array}$ & $\begin{array}{l}\text { DMSO/EmimOAc } \\
\text { (wt ratio) }\end{array}$ & DS values $^{b}$ \\
\hline 10 & 4000 & 33.3 & 1.1 & 2.96 \\
\hline 11 & 1100 & 9.2 & 4.0 & 2.99 \\
\hline 12 & 630 & 5.3 & 7.0 & 2.98 \\
\hline 13 & 400 & 3.3 & 11 & 2.96 \\
\hline 14 & 300 & 2.5 & 15 & 2.92 \\
\hline 15 & 150 & 1.3 & 29 & 2.78 \\
\hline
\end{tabular}

${ }^{a}$ The reaction conditions for the transesterification reactions of cellulose with IPA are as follows; EmimOAc as a solvent and an organocatalyst; the reaction was conducted for 18 hours; $4 \mathrm{~mL}$ of DMSO was used as a co-solvent; $120 \mathrm{mg}$ of cellulose was used as a starting compound; initial [IPA]/[OH] was adjusted to be 16/1; Ar atmosphere. ${ }^{b}$ Determined by ${ }^{1} \mathrm{H}$ NMR measurements in DMSO- $d_{6}$.

expensive. In addition, as noted above, the protocol used there requires rather high reactant loadings when compared to acylation reactions with activated carbonyl compounds such as acid chlorides and acid anhydrides. Thus, the target of the optimization of the IL-catalyzed transesterification of cellulose was to reduce the required amount of both the ILs and reactants while maintaining the high conversion rate. For this, a mixed solvent system composed of EmimOAc and DMSO was employed not only because DMSO addition could accelerate the transesterification reactions but also because DMSO has been known not to disturb cellulose dissolution processes due to its high polarity..$^{37,39,40}$ Cellulose modification reactions in the DMSO/ EmimOAc mixed solvent system was conducted by varying the DMSO/EmimOAc mixing ratio (Table 3). As a reference, DMSO was used as a co-solvent while keeping the originally reported

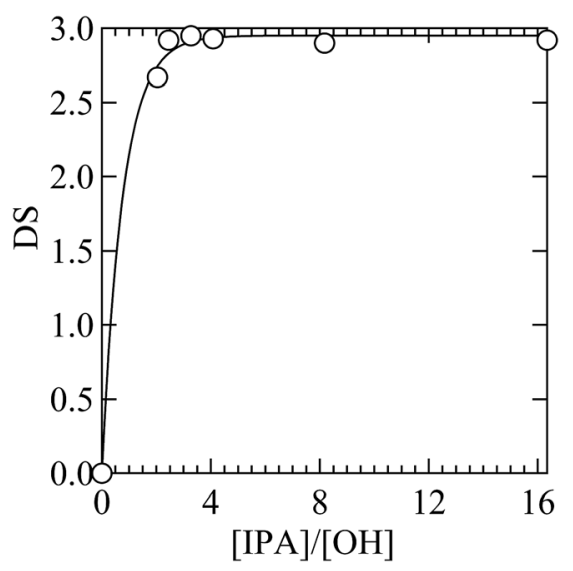

Fig. 4 Effect of the IPA-to-hydroxy-group ratio on the transesterification reaction efficiency in EmimOAc/DMSO mixed solvent systems (line; guidance). The reaction conditions are as follows; EmimOAc as a solvent and an organocatalyst; EmimOAc/cellulose (wt ratio) $=2.5 ; 4 \mathrm{~mL}$ of DMSO was used as a co-solvent; Ar atmosphere; the reactions were conducted for 18 hours. reaction conditions (run 10, Table 3). It is remarkable that compared to this reference, we were able to reduce EmimOAc by $93 \%$ while maintaining the high DS value $(\sim 2.9)$ for the cellulose acetate product (run 14, Table 3). Along with a successful reduction in the amount of ILs, we were able to reduce the reactant loading markedly (Fig. 4). The initial IPA concentration was reduced by $85 \%$ while maintaining the high DS value $(\sim 2.9)$ for cellulose acetate. ${ }^{\mathbf{4 1}}$ The combined experimental/theoretical analysis presented here demonstrates that mechanistic understanding could play a very useful and important role in the design and optimization of reaction systems.

\section{Conclusions}

We provided molecular insights into the organocatalytic properties of imidazolium salts for transesterification reactions. Experimental and computational analysis of a model reaction system indicates that transesterification is most probably catalysed by the anionic species of ILs. The mechanistic consideration of IL-catalyzed reactions successfully led to a positive cosolvent effect on cellulose modification reactions in IL-based solvent systems. More broadly, the mechanistic insights obtained here for Im-ILs will be very useful in molecular design of next-generation of organocatalysts.

\section{Experimental section}

\section{Materials}

EmimOAc (95\%) was available from Kanto Chemical Co., Inc., and used without further purification. IPA was available from the Sigma-Aldrich Chemicals Co., and used as received. As a cellulose source, Avicel PH-101 was purchased from SigmaAldrich Chemicals Co. BiPrimCl, ${ }^{42}$ EMPhimBr $^{43}$ and EMBnimBr ${ }^{44}$ were synthesized according to reported protocols. The apparent molecular weight of the avicel was determined by size exclusion chromatography (SEC) measurements in THF with 
polystyrene calibrations of carbanilated cellulose sample (reacted with phenyl isocyanate). The carbanilation reaction was carried out essentially according to a previously reported method. ${ }^{45}$ The apparent number average degree of polymerization of original cellulose was calculated to be 105. All other chemicals were commercially available and used without further purification unless otherwise stated.

\section{Instruments}

All the ${ }^{1} \mathrm{H}$ and ${ }^{13} \mathrm{C}$ NMR spectra in solution were recorded by JEOL 400 and $600 \mathrm{MHz}$ FT-NMR spectrometers in deuterated solvents, and the chemical shifts $(\delta)$ were given in ppm with either the solvent peak or TMS as the internal standard. The IR spectra were recorded by a Thermo Fisher Scientific Nicolet iS10 equipped with an ATR unit.

\section{Experimental procedures}

Synthesis of various imidazolium salts. In principle, all imidazolium salts were prepared based on the previously reported ion-exchange method starting from corresponding halide salts. ${ }^{46}$ Although synthesis of EDmimOAc was already reported, a synthetic protocol for the ion-exchange was slightly modified according to the previously reported method. ${ }^{\mathbf{4 6}}$ Thus, synthesis of EDmimOAc and BiPrimOAc was also briefly included.

Typical procedure for ion-exchange protocol to produce acetate-based imidazolium salts starting from corresponding halide salts. As an example, synthesis of 1-methyl-2-phenyl-3ethyl imidazolium acetate (EMPhimOAc) is described: under open conditions, a water/MeOH solution containing acetic acid (water $/ \mathrm{MeOH} / \mathrm{AcOH}=50 / 50 / 1, \mathrm{v} / \mathrm{v} / \mathrm{v}$ ) was passed through a column filled with Amberlyst A26 hydroxide form. The excess $\mathrm{AcOH}$ in the column was removed by passing through water/ $\mathrm{MeOH}$ solutions. Then, a $200 \mathrm{~mL} \mathrm{MeOH}$ solution of 1-methyl2-phenyl-3-ethyl imidazolium bromide (3.0 g, $11.9 \mathrm{mmol})$ was passed though the acetate anion trapped column. The combined eluent was dried under vacuum conditions at $70{ }^{\circ} \mathrm{C}$ overnight to afford a slightly colored solid. Yield: $2.76 \mathrm{~g}(94.7 \%)$. ${ }^{1} \mathrm{H}$ NMR $\left(400 \mathrm{MHz}, \mathrm{DMSO}-d_{6}\right) \delta ; 8.18(\mathrm{~d}, J=2.1 \mathrm{~Hz}, 1 \mathrm{H}), 8.14(\mathrm{~d}$, $J=2.1 \mathrm{~Hz}, 1 \mathrm{H}), 7.91-7.52(\mathrm{~m}, 5 \mathrm{H}), 4.02(\mathrm{q}, J=7.3 \mathrm{~Hz}, 2 \mathrm{H}), 3.68$ $(\mathrm{s}, 3 \mathrm{H}), 1.52(\mathrm{~s}, 3 \mathrm{H}), 1.27(\mathrm{t}, J=7.2 \mathrm{~Hz}, 3 \mathrm{H}) .{ }^{13} \mathrm{C} \mathrm{NMR}(100 \mathrm{MHz}$, DMSO- $\left.d_{6}\right) \delta ; 172.39,143.64,132.30,130.55,129.52,123.81$, 121.62, 121.46, 43.54, 35.43, 26.30, 15.07 .

Synthesis of EMBnimOAc. The synthesis of EMBnimOAc was started from corresponding bromide salt. The ion-exchange protocol was the same as the EMPhimOAc synthesis. Yield: $1.80 \mathrm{~g}(98.0 \%) .{ }^{1} \mathrm{H}$ NMR (400 MHz, DMSO- $\left.d_{6}\right) \delta 10.80(\mathrm{~s}, 1 \mathrm{H})$, 8.13-7.97 (m, 2H), 7.77-7.55 (m, 2H), $4.55(\mathrm{q}, J=7.3 \mathrm{~Hz}, 3 \mathrm{H})$, $4.11(\mathrm{~s}, 3 \mathrm{H}), 1.62(\mathrm{~s}, 3 \mathrm{H}), 1.52(\mathrm{t}, J=7.2 \mathrm{~Hz}, 3 \mathrm{H}) .{ }^{13} \mathrm{C}$ NMR $(100$ MHz, DMSO- $\left.d_{6}\right) \delta ; 173.38,144.03,131.95,130.81,126.26$, 113.53, 113.44, 41.76, 32.96, 26.12, 14.37 .

Synthesis of BiPrimOAc. The synthesis of BiPrimOAc was started from corresponding chloride salt. The ion-exchange protocol was the same as the EMPhimOAc synthesis. Yield: $2.08 \mathrm{~g}(98.9 \%) .{ }^{1} \mathrm{H}$ NMR (400 MHz, DMSO- $\left.d_{6}\right) \delta 10.61(\mathrm{~s}, 1 \mathrm{H})$, $8.62(\mathrm{~d}, J=1.6 \mathrm{~Hz}, 2 \mathrm{H}), 7.68(\mathrm{t}, J=7.8 \mathrm{~Hz}, 2 \mathrm{H}), 7.52(\mathrm{~d}, J=$
$7.8 \mathrm{~Hz}, 4 \mathrm{H}), 2.43-2.30(\mathrm{~m}, 4 \mathrm{H}), 1.32(\mathrm{~s}, 3 \mathrm{H}), 1.26(\mathrm{~d}, J=6.8 \mathrm{~Hz}$, $12 \mathrm{H}), 1.17$ (d, $J=6.9 \mathrm{~Hz}, 12 \mathrm{H}) .{ }^{13} \mathrm{C}$ NMR (100 MHz, DMSO- $\left.d_{6}\right) \delta$; 172.19 , 144.83, 140.10, 131.75, 130.22, 126.21, 124.56, 28.65, 25.91, 24.11, 23.14 .

Synthesis of EDmimOAc. The synthesis of EDmimOAc was started from corresponding bromide salt. The ion-exchange protocol was the same as the EMPhimOAc synthesis. Yield: $1.72 \mathrm{~g}(96.0 \%) .{ }^{1} \mathrm{H}$ NMR (400 MHz, DMSO- $\left.d_{6}\right) \delta ; 7.85(\mathrm{~d}, J=$ $2.1 \mathrm{~Hz}, 1 \mathrm{H}), 7.83(\mathrm{~d}, J=2.1 \mathrm{~Hz}, 1 \mathrm{H}), 4.18(\mathrm{q}, J=7.3 \mathrm{~Hz}, 2 \mathrm{H}), 3.78$ $(\mathrm{s}, 3 \mathrm{H}), 2.61(\mathrm{~s}, 3 \mathrm{H}), 1.50(\mathrm{~s}, 3 \mathrm{H}), 1.31(\mathrm{t}, J=7.3 \mathrm{~Hz}, 3 \mathrm{H}) .{ }^{13} \mathrm{C}$ NMR $\left(100 \mathrm{MHz}, \mathrm{DMSO}-d_{6}\right) \delta ; 172.54,144.01,122.60,120.53$, 42.69, 34.54, 26.27, 14.96, 9.02.

Transesterification reaction of 2-PA with IPA catalyzed by imidazolium salts. A typical transesterification reaction of 2-PA was carried out as follows (run 1, Table 1): under Ar atmosphere, a solution of 2-PA (1.0 mL, $8.4 \mathrm{mmol})$, IPA (1.4 mL, $12.9 \mathrm{mmol})$, and EmimOAc (143 mg, $0.84 \mathrm{mmol}$ ) was stirred for 16 hours under Ar conditions at $80{ }^{\circ} \mathrm{C}$. After the reaction mixture was exposed to the air, a small portion of reaction mixture was taken for NMR measurements in order to determine the reaction conversions. The reaction mixture was diluted with $\mathrm{CH}_{2} \mathrm{Cl}_{2}$ and the organic layer was washed with water. The combined organic layer was dried with $\mathrm{Na}_{2} \mathrm{SO}_{4}$ the product was purified by column chromatography (silica gel, $\mathrm{CH}_{2} \mathrm{Cl}_{2} /$ hexane $=1 / 1$ ) to yield a colourless oil. Yield: $763 \mathrm{mg}$ (55.7\%).

Transesterification reaction of cellulose in EmimOAc/DMSO mixed solvents. A typical reaction based on the transesterification of cellulose was carried out as follows: a solution of cellulose $\left.(120 \mathrm{mg} \text {, [monomeric unit }]_{0}=0.74 \mathrm{mmol}\right)$ in EmimOAc ( $4.0 \mathrm{~g}$ ) was dried overnight under vacuum conditions at $80{ }^{\circ} \mathrm{C}$. After the drying process was finished, DMSO $(4 \mathrm{~mL})$ and IPA ( $4 \mathrm{~mL}$ ) was added under an Ar atmosphere. After the reaction mixture was stirred for 18 hours, the reaction mixture was diluted with $\mathrm{CH}_{2} \mathrm{Cl}_{2}$ and poured into a large amount of $\mathrm{MeOH}$. The polymer was purified by reprecipitation $\left(\mathrm{CH}_{2} \mathrm{Cl}_{2} / \mathrm{MeOH}\right)$ to yield a pale yellowish powder. Yield: $170 \mathrm{mg}$ (79.7\%).

Determination of DS values of cellulose acetates. The DS values of the cellulose acetate was determined by ${ }^{1} \mathrm{H}$ NMR measurements of the cellulose derivatives that were obtained by the reaction of cellulose acetate with an excess amount of benzoyl chloride. The typical procedure is as follows: a $4 \mathrm{~mL}$ $\mathrm{CHCl}_{3}$ solution of the cellulose acetate $(100 \mathrm{mg}, 347 \mu \mathrm{mol})$, benzoyl chloride (440 mg, $3.1 \mathrm{mmol}$ ) and triethylamine $(318 \mathrm{mg}$, $3.1 \mathrm{mmol}$ ) was stirred for 24 hours at r.t. The reaction mixture was then poured into a large amount of $\mathrm{MeOH}$. The obtained precipitate was collected and dried under vacuum conditions. Isolated yield: $90.4 \mathrm{mg}(66.2 \%)$.

The DS values were calculated using the following equation:

$$
\mathrm{DS}=3 \times \frac{\text { peak area }}{1.5-2.3 \mathrm{ppm} / 3} \frac{\text { prea }_{7-8.5 \mathrm{ppm}} / 5}{\text { peak } \text { area }_{1.5-2.3 \mathrm{ppm}} / 3+\text { peak }}
$$

In order to avoid potential overlapping of the aromatic protons and solvent peak, NMR measurements were conducted in DMSO- $d_{6}$. 
DFT calculations. DFT calculations were performed to investigate possible mechanisms of the transesterification reaction promoted by imidazolium-based ionic liquids. The Synchronous Transit-Guided Quasi-Newton (STQN) method is used for locating of transition states. ${ }^{47,48}$ The partial atomic charges are determined with the electro-static potential (ESP) method. ${ }^{49-51}$ The Gaussian 09 package is used for geometry optimizations with the B3LYP hybrid density functional and 6$31 \mathrm{G}(\mathrm{d}, \mathrm{p})$ basis sets. ${ }^{52-55}$

\section{Acknowledgements}

This research was promoted by COI program Construction of next-generation infrastructure using innovative materials Realization of a safe and secure society that can coexist with the Earth for centuries $\sim$ supported by MEXT and JST. This study was also supported in part by an Advanced Low Carbon Technology Research and Development Program (ALCA) of the JST and the Cross-ministerial Strategic Innovation Promotion Program (SIP) from the JST. This work was also supported in part by National Science Foundation through NSF Grant No. CHE-1223988.

\section{Notes and references}

1 H. Kopetz, Nature, 2013, 494, 29-31.

2 R. Rinaldi, Angew. Chem., Int. Ed., 2014, 53, 8559-8560.

3 R. P. Swatloski, S. K. Spear, J. D. Holbrey and R. D. Rogers, J. Am. Chem. Soc., 2002, 124, 4974-4975.

4 M. Gericke, P. Fardim and T. Heinze, Molecules, 2012, 17, 7458-7502.

5 A. Pinkert, K. N. Marsh, S. Pang and M. P. Staiger, Chem. Rev., 2009, 109, 6712-6728.

6 A. Jordan and N. Gathergood, Chem. Soc. Rev., 2015, 44, 8200-8237.

7 N. V. Plechkova and K. R. Seddon, Chem. Soc. Rev., 2008, 37, 123-150.

8 H. Wang, G. Gurau and R. D. Rogers, Chem. Soc. Rev., 2012, 41, 1519-1537.

9 F. Zhou, Y. Liang and W. Liu, Chem. Soc. Rev., 2009, 38, 25902599.

10 W. Miao and T. H. Chan, Acc. Chem. Res., 2006, 39, 897-908.

11 J. Dupont, R. F. de Souza and P. A. Z. Suarez, Chem. Rev., 2002, 102, 3667-3692.

12 Q. Zhang and J. N. M. Shreeve, Chem. Rev., 2014, 114, 1052710574.

13 T. Welton, Coord. Chem. Rev., 2004, 248, 2459-2477.

14 T. Liebert, Macromol. Symp., 2008, 262, 28-38.

15 M. T. Clough, K. Geyer, P. A. Hunt, S. Son, U. Vagt and T. Welton, Green Chem., 2015, 17, 231-243.

16 G. Ebner, S. Schiehser, A. Potthast and T. Rosenau, Tetrahedron Lett., 2008, 49, 7322-7324.

17 O. A. El Seoud, A. Koschella, L. C. Fidale, S. Dorn and T. Heinze, Biomacromolecules, 2007, 8, 2629-2647.

18 S. Zhu, Y. Wu, Q. Chen, Z. Yu, C. Wang, S. Jin, Y. Ding and G. Wu, Green Chem., 2006, 8, 325-327.
19 H. Zhang, J. Wu, J. Zhang and J. He, Macromolecules, 2005, 38, 8272-8277.

20 R. Kakuchi, M. Yamaguchi, T. Endo, Y. Shibata, K. Ninomiya, T. Ikai, K. Maeda and K. Takahashi, RSC Adv., 2015, 5, 72071-72074.

21 D. M. Flanigan, F. Romanov-Michailidis, N. A. White and T. Rovis, Chem. Rev., 2015, 115, 9307-9387.

22 M. N. Hopkinson, C. Richter, M. Schedler and F. Glorius, Nature, 2014, 510, 485-496.

23 H. Rodriguez, G. Gurau, J. D. Holbrey and R. D. Rogers, Chem. Commun., 2011, 47, 3222-3224.

24 D. Liu, Y. Zhang and E. Y. X. Chen, Green Chem., 2012, 14, 2738-2746.

25 R. Lambert, P. Coupillaud, A. L. Wirotius, J. Vignolle and D. Taton, Macromol. Rapid Commun., 2016, 37, 1143-1149.

26 L. F. Zhang, X. L. Fu and G. H. Gao, ChemCatChem, 2011, 3, 1359-1364.

27 M. Selva, M. Noe, A. Perosa and M. Gottardo, Org. Biomol. Chem., 2012, 10, 6569-6578.

28 S. R. Labafzadeh, K. J. Helminen, I. Kilpelainen and A. W. King, ChemSusChem, 2015, 8, 77-81.

29 After a column purification, the product was obtained in $55.7 \%$ isolated yield.

30 F. G. Bordwell, Acc. Chem. Res., 1988, 21, 456-463.

31 Z. Kelemen, O. Holloczki, J. Nagy and L. Nyulaszi, Org. Biomol. Chem., 2011, 9, 5362-5364.

32 G. Gurau, H. Rodríguez, S. P. Kelley, P. Janiczek, R. S. Kalb and R. D. Rogers, Angew. Chem., Int. Ed., 2011, 50, 12024-12026.

33 M. Besnard, M. I. Cabaco, F. V. Chavez, N. Pinaud, P. J. Sebastiao, J. A. P. Coutinho and Y. Danten, Chem. Commun., 2012, 48, 1245-1247.

34 F. Yan, N. R. Dhumal and H. J. Kim, Phys. Chem. Chem. Phys., 2017, 19, 1361-1368.

35 S. Naumann, A. W. Thomas and A. P. Dove, ACS Macro Lett., 2016, 5, 134-138.

36 S. Naumann, A. W. Thomas and A. P. Dove, Angew. Chem., Int. Ed., 2015, 54, 9550-9554.

37 A. Xu, Y. Zhang, Y. Zhao and J. Wang, Carbohydr. Polym., 2013, 92, 540-544.

38 W. R. Fawcett, P. Brooksby, D. Verbovy, I. Bakó and G. Pálinkás, J. Mol. Liq., 2005, 118, 171-178.

39 R. Rinaldi, Chem. Commun., 2011, 47, 511-513.

40 J.-M. Andanson, E. Bordes, J. Devemy, F. Leroux, A. A. H. Padua and M. F. C. Gomes, Green Chem., 2014, 16, 2528-2538.

41 A kinetic investigation on the cellulose modification reaction under the optimized condition was included in Fig. S1 in ESI. $\dagger$

42 T. Fujimoto and T. Ritter, Org. Lett., 2015, 17, 544-547.

43 B. Lin, H. Dong, Y. Li, Z. Si, F. Gu and F. Yan, Chem. Mater., 2013, 25, 1858-1867.

44 M. J. Saif and K. R. Flower, Transition Met. Chem., 2012, 38, 113-118.

45 K. Schlufter, H.-P. Schmauder, S. Dorn and T. Heinze, Macromol. Rapid Commun., 2006, 27, 1670-1676.

46 E. Alcalde, I. Dinares, A. Ibanez and N. Mesquida, Molecules, 2012, 17, 4007-4027. 
47 C. Peng and H. Bernhard Schlegel, Isr. J. Chem., 1993, 33, 449-454.

48 C. Peng, P. Y. Ayala, H. B. Schlegel and M. J. Frisch, J. Comput. Chem., 1996, 17, 49-56.

49 A. D. Becke, Phys. Rev. A, 1988, 38, 3098-3100.

50 C. M. Breneman and K. B. Wiberg, J. Comput. Chem., 1990, 11, 361-373.

51 C. I. Bayly, P. Cieplak, W. Cornell and P. A. Kollman, J. Phys. Chem., 1993, 97, 10269-10280.

52 A. D. Becke, J. Chem. Phys., 1993, 98, 5648-5652.

53 J. C. Burant, G. E. Scuseria and M. J. Frisch, J. Chem. Phys., 1996, 105, 8969-8972.

54 A. Laio, J. VandeVondele and U. Rothlisberger, J. Phys. Chem. $B, 2002$, 106, 7300-7307.

55 M. J. Frisch, G. W. Trucks, H. B. Schlegel, G. E. Scuseria, M. A. Robb, J. R. Cheeseman, G. Scalmani, V. Barone,
B. Mennucci, G. A. Petersson, H. Nakatsuji, M. Caricato, X. Li, H. P. Hratchian, A. F. Izmaylov, J. Bloino, G. Zheng, J. L. Sonnenberg, M. Hada, M. Ehara, K. Toyota, R. Fukuda, J. Hasegawa, M. Ishida, T. Nakajima, Y. Honda, O. Kitao, H. Nakai, T. Vreven, J. A. Montgomery Jr, J. E. Peralta, F. Ogliaro, M. Bearpark, J. J. Heyd, E. Brothers, K. N. Kudin, V. N. Staroverov, R. Kobayashi, J. Normand, K. Raghavachari, A. Rendell, J. C. Burant, S. S. Iyengar, J. Tomasi, M. Cossi, N. Rega, J. M. Millam, M. Klene, J. E. Knox, J. B. Cross, V. Bakken, C. Adamo, J. Jaramillo, R. Gomperts, R. E. Stratmann, O. Yazyev, A. J. Austin, R. Cammi, C. Pomelli, J. W. Ochterski, R. L. Martin, K. Morokuma, V. G. Zakrzewski, G. A. Voth, P. Salvador, J. J. Dannenberg, S. Dapprich, A. D. Daniels, Ö. Farkas, J. B. Foresman, J. V. Ortiz, J. Cioslowski, and D. J. Fox, Gaussian 09, Gaussian, Inc., Wallingford CT, 2009. 